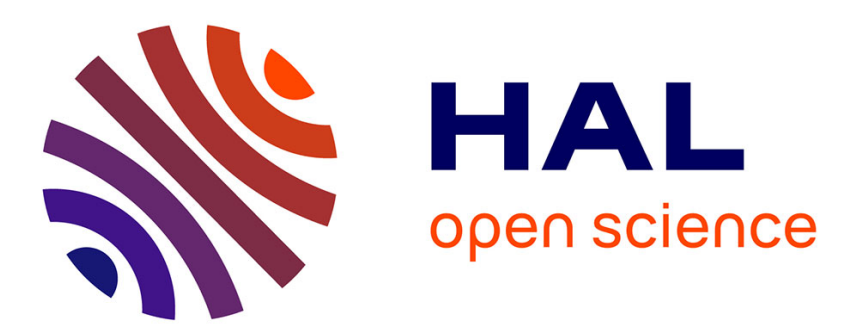

\title{
Propriétés optiques déduites du spectre de réflexion de CuI à basse température
}

\author{
C. Jung, S. Lewonczuk, J. Ringeissen, S. Nikitine
}

\section{To cite this version:}

C. Jung, S. Lewonczuk, J. Ringeissen, S. Nikitine. Propriétés optiques déduites du spectre de réflexion de $\mathrm{CuI}$ à basse température. Revue de Physique Appliquée, 1972, 7 (1), pp.43-48. 10.1051/rphysap:019720070104300 . jpa-00243595

\section{HAL Id: jpa-00243595 https://hal.science/jpa-00243595}

Submitted on 1 Jan 1972

HAL is a multi-disciplinary open access archive for the deposit and dissemination of scientific research documents, whether they are published or not. The documents may come from teaching and research institutions in France or abroad, or from public or private research centers.
L'archive ouverte pluridisciplinaire HAL, est destinée au dépôt et à la diffusion de documents scientifiques de niveau recherche, publiés ou non, émanant des établissements d'enseignement et de recherche français ou étrangers, des laboratoires publics ou privés. 


\title{
PROPRIÉTÉS OPTIQUES \\ DÉDUITES DU SPECTRE DE RÉFLEXION DE CuI A BASSE TEMPÉRATURE
}

\section{JUNG, S. LEWONCZUK, J. RINGEISSEN et S. NIKITINE}

Laboratoire de Spectroscopie et d'Optique du Corps Solide (*), Institut de Physique, Université Louis Pasteur, Strasbourg

(Reçu le 22 novembre 1971)

\begin{abstract}
Résumé. - Le spectre de réflexion d'un cristal massif de CuI a été étudié aux basses températures. Le spectre d'absorption en a été déduit par une analyse de Kramers-Krönig. Un ordre de grandeur des facteurs $f$, en accord qualitatif avec des transitions excitoniques de première classe, a été obtenu. On observe trois niveaux excitoniques formés avec les trous de la bande $\Gamma_{8}$ et deux avec des trous de $\Gamma_{7}$. Le niveau $n=1$ de $\Gamma_{8}$ présente une structure fine qui peut être expliquée dans le couplage $\mathrm{JJ}$ et, par analogie avec $\mathrm{CuCl}$ et $\mathrm{CuBr}$, un très faible maximum d'absorption peut être attribué à une transition assistée d'un phonon longitudinal.

Du côté des plus grandes énergies, on a observé trois transitions liées à des bandes plus profondes. Ces transitions s'interprètent en première approximation à partir d'un schéma de bandes extrapolé à partir de $\mathrm{CuCl}$.
\end{abstract}

\begin{abstract}
The reflection spectrum on a CuI bulk crystal has been studied at low temperatures and the absorption spectrum has been deduced by means of a Kramers-Krönig analysis. Approximate values of the oscillator strengths $f$ have been calculated. They are in qualitative agreement with first class excitonic transitions. Three excitonic levels have been observed with holes formed in the $\Gamma_{8}$ band and two with holes from $\Gamma_{7}$. The $n=1$ level of $\Gamma_{8}$ shows a fine structure which can be explained in the $\mathrm{JJ}$ coupling scheme. As in $\mathrm{CuCl}$ and $\mathrm{CuBr}$, a very weak absorption peak of $\mathrm{CuI}$ can be assigned to a transition accompanied by a longitudinal phonon. On the higher energy side, transitions due to deeper bands have been observed. These transitions have been interpreted by extrapolation from a $\mathrm{CuCl}$ band scheme.
\end{abstract}

Introduction. - L'iodure cuivreux a déjà fait l'objet de nombreuses études portant sur les spectres d'absorption, de luminescence et de réflexion, à basse température, au voisinage de l'absorption fondamentale.

Il a été un des premiers composés pour lequel on a cité un spectre excitonique de première classe [1]. Par rapport à $\mathrm{CuCl}$ et $\mathrm{CuBr}$, ces raies excitoniques observées en absorption présentaient pourtant la particularité d'apparaître en doublet. Ce dédoublement apparaissait aussi dans le spectre de réflexion de couches minces [2].

D'autre part, ces spectres de réflexion se sont avérés très sensibles à la nature de la lame support [3].

Enfin, il faut noter que les spectres d'absorption à $4{ }^{\circ} \mathrm{K}$ sont très sensibles à la méthode de préparation des couches minces [4]. Par exemple, Cardona cite des raies qui sont, soit liées à la présence de phase wurtzite, soit encore à une structure hexagonale particulière aux couches minces [5]. De telles raies

$\left({ }^{*}\right)$ Groupe de recherche $\mathrm{n}^{\circ} 15$ du CNRS. n'ont pas été observées sur couches minces recristallisées [4].

Ces particularités méritaient d'être examinées dans un cadre plus large : l'étude systématique des composés I.VII qui cristallisent dans la structure zincblende. En effet, dans les dernières années, nous avons consacré un certain nombre de travaux à $\mathrm{CuCl}$ et $\mathrm{CuBr}$ portant essentiellement sur les propriétés excitoniques [6], [7].

L'étude de CuI présente un autre intérêt. Song [8] a proposé un schéma de bandes de CuI extrapolé à partir de $\mathrm{CuCl}$, pour lequel la structure de bande a été calculée. Dans ce schéma, il apparaît que, dans le cas spécifique de $\mathrm{CuI}$, un certain nombre d'énergies interbandes se situent dans le domaine de l'UV proche $(\lambda>1900 \AA)$.

Pour approfondir ces différents points, nous nous sommes proposés de mesurer, à basse température, le pouvoir réflecteur de cristaux massifs de CuI, entre $6000 \AA$ et $1900 \AA$.

Dans ce travail, on se limite aux propriétés optiques liées aux excitons intrinsèques. Les raies d'absorption ou de luminescence liées à l'existence de complexes 
excitoniques ne seront donc pas discutées. Ces spectres ont déjà fait l'objet de nombreuses études [9], [1].

Montage expérimental. - Le montage photométrique à double faisceau comporte un monochromageur Jarrel Ash (Ebert 0,5 m), éclairé par une lampe XBO $450 \mathrm{~W}$. La bande passante est de l'ordre de $1 \AA$ dans la région spectrale qui concerne la première série excitonique $(4000 \AA)$; elle peut atteindre 5 à $6 \AA$ vers $2000 \AA$. Les photomultiplicateurs sont du type EMI $9526 \mathrm{KS}$. Les signaux électriques analogiques sont envoyés, d'une part sur un enregistreur Méci monté en détection de rapport, d'autre part ils sont digitalisés pour l'exploitation sur ordinateur.

L'échantillon est refroidi sous vide, par conduction métallique dans un cryostat TBT à une température de l'ordre de 8 à $9{ }^{\circ} \mathrm{K}$.

Le pouvoir réflecteur absolu est évalué en un point où l'indice est connu. A la température de $4,2 \circ \mathrm{K}$, par l'étude des sectres cannelés, Reiss donne une valeur de 2,55 [1] à $23000 \mathrm{~cm}^{-1}$.

Résultats expérimentaux. - Les résultats obtenus sont illustrés par la figure 1. Il apparaît d'abord deux rayons restants intenses $v_{1 \mathrm{~d}}$ et $v_{1 \mathrm{f}}(*)$. La raie $v_{1 \mathrm{~d}}$ présente une raie de structure fine $v_{\mathrm{J}}$. Au voisinage immédiat de $v_{1 \mathrm{~d}}$ (Fig. 1), on observe trois anomalies de réflexion, désignées respectivement par $v_{2 \mathrm{~d}}, v_{3 \mathrm{~d}}$ et $v_{2 \mathrm{~s}}$ (Fig. 1). De même, la bande $v_{1 \mathrm{f}}$ est accompagnée d'une anomalie de réflexion $v_{2 \mathrm{f}}$ d'amplitude très faible, de l'ordre de $1 \%$ seulement (Fig. 1). En allant vers

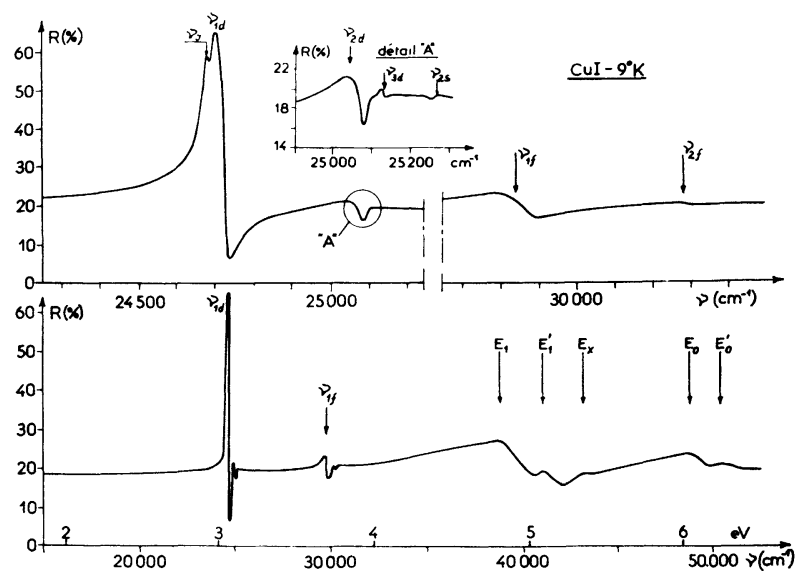

FIg. 1. - Spectre de réflexion de CuI.

les grandes énergies, le pouvoir réflecteur augmente d'abord, puis il présente cinq maxima ou paliers que nous désignons en allant vers les grandes énergies $\operatorname{par} E_{1}, E_{1}^{\prime}, E_{x}, E_{0}, E_{0}^{\prime}$.

$\left({ }^{*}\right) v_{1 \mathrm{~d}}$ désigne une transition de création d'un exciton formé par un électron de la bande de conduction avec un trou de symétrie $\Gamma_{8}, v_{1 \mathrm{f}}$ une transition lors de la formation d'un exciton avec un trou $\Gamma_{7}$
Mesure quantitative du spectre d'absorption. Discussion. - L'étude du profil des raies d'absorption excitoniques de première classe présente des difficultés de principe. Elle peut se faire selon deux méhodes : la mesure directe sur couche mince ou le calcul de $\mathrm{k}$ et $\mathrm{n}$ par la transformation de KramersKrönig du spectre de réflexion.

a) Le coefficient d'absorption pouvant atteindre des valeurs de l'ordre de $10^{6} \mathrm{~cm}^{-1}$ au sommet d'une raie excitonique, une précision acceptable des mesures photométriques suppose une épaisseur de l'ordre de $10^{2} \AA$. Dans ce cas, la grosseur des grains n'est plus contrôlable et l'on sait que, pour des grains de l'ordre de $100 \AA$ à $200 \AA$, le spectre excitonique disparaît pratiquement [10]. A cet effet, pour CuI s'ajoute le dédoublement des raies, qui est dû probablement aux contraintes de la lame support.

b) Devant ces difficultés, on est tenté de déterminer $\mathrm{K}$ à partir de la réflexion d'un gros cristal par la transformation de Kramers-Krönig. Toutefois, celle-ci est quelque peu incertaine. Cela tient d'abord au fait que l'intégrale est toujours calculée pour un intervalle relativement restreint; nous reviendrons sur ce point plus loin.

D'autre part, l'opinion a été exprimée que la transformée KK ne peut être appliquée sans précautions dans une raie excitonique, du fait de la dispersion spatiale de Pekar [11], d'après laquelle la théorie classique de l'électromagnétisme n'est plus valable.

Toutefois, les corrections à apporter, qui sont à l'étude, n'ont pas encore été explicitées. Nous assimilerons donc l'exciton à un oscillateur classique qui peut être traité par une transformée $\mathrm{KK}$.

Analyse Kramers-Krönig du spectre de réflexion. Facteurs F. - Dans le cas spécifique de CuI, fcette analyse est particulièrement délicate. Bien que le pouvoir réflecteur ait été déterminé dans un grand domaine spectral $\left(16000\right.$ à $\left.52000 \mathrm{~cm}^{-1}\right)$, il s'avère que, du côté des grandes énergies, la dernière valeur mesurée de $R$ est encore notablement plus élevée que celle du côté des petites énergies. De ce fait, la borne supérieure de l'intégrale se situe dans une région où l'absorption est toujours importante, ce qui suppose un déphasage important entre l'onde incidente et l'onde réfléchie.

En plus, la réflexion spéculaire mesurée dans l'UV donne certainement une valeur par défaut, puisque le taux de lumière diffusé par l'échantillon augmente en allant vers les courtes longueurs d'onde. A cela s'ajoute l'indétermination qui peut provenir du fait que le montage vers $2000 \AA$ est à la limite extrême de ses possibilités.

Il n'est alors pas étonnant que le calcul du déphasage à partir de l'intégrale KK, sans autre hypothèse quant aux valeurs aux bornes, puisse conduire à un profil aberrant des raies excitoniques.

Sans pour autant éliminer la possibilité d'erreur 
portant sur le pouvoir réflecteur, nous admettrons que ce profil aberrant est essentiellement dû à la non, connaissance du spectre au-delà des deux bornes (principalement du côté des grandes énergies) ou, en d'autres termes, que l'exploration du spectre n'est pas assez étendue pour isoler la seule contribution des bandes observées.

On sait que pour la résolution de l'intégrale, la courbe $R(v)$ est partagée en segments dont la somme des pentes, chacune affectée du facteur de poids correspondant, donne en tout point la valeur de la phase [12].

Nous admettrons que les pentes des deux segments extrêmes $\mathrm{p}_{1}$ et $\mathrm{p}_{2}$ peuvent traduire globalement, avec le facteur de poids correspondant, la contribution à la phase en un point donné des bandes d'absorption situées en dehors de la plage du spectre de réflexion. On peut donc espérer trouver un couple de valeurs $p_{1}$ et $p_{2}$ qui, à partir du pouvoir réflecteur mesuré entre deux bornes, donne une courbe d'absorption physiquement acceptable.

Pour la détermination des paramètres $p_{1}$ et $p_{2}$, les critères suivants ont été retenus : vers $16000 \mathrm{~cm}^{-1}$, le coefficient d'absorption $K$ doit être aussi faible que possible ; entre ce point et la raie $v_{1 \mathrm{~d}}, K$ ne peut passer par un minimum. Les calculs effectués sur ordinateur ont permis d'obtenir le tracé $K(v)$ optimum représenté par la figure 2 .

Les remarques suivantes s'imposent : l'approximation faite dans le calcul est probablement valable

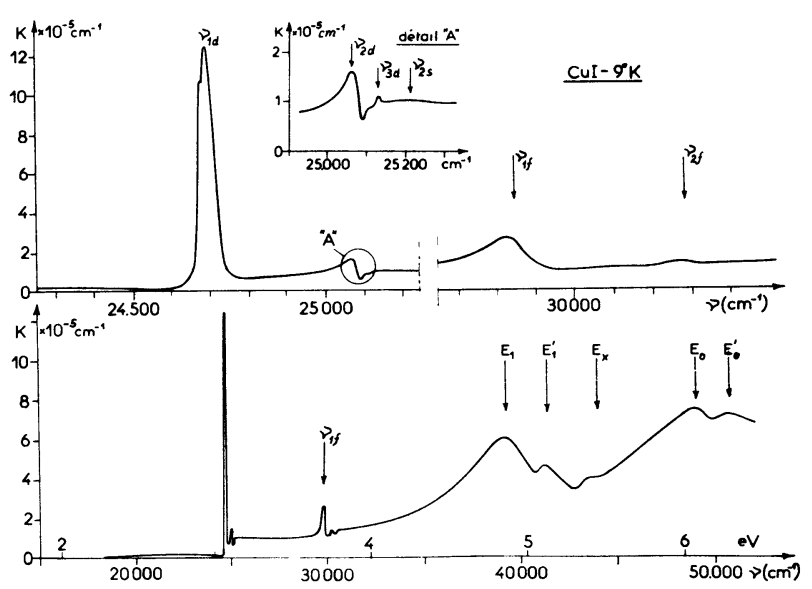

FIG. 2. - Spectre d'absorption de CuI calculé à partir d'une analyse KK du spectre de réflexion. pour les raies situées loin de l'extrémité du spectre étudié ; par contre, le profil de l'absorption ainsi obtenu vers $50000 \mathrm{~cm}^{-1}$ doit être considéré avec beaucoup de prudence.

On peut toutefois noter que les positions spectrales des maxima d'absorption ainsi calculées correspondent assez bien aux maxima de réflexion.

Par ailleurs, on peut noter que les maxima d'absorption sont en excellent accord avec les positions spectrales des composantes de grande énergie des doublets observés en absorption sur une couche mince (tableau I).

\section{TABLEAU I}

Tableau comparatif de la position des raies d'absorption

\begin{tabular}{cllr}
$v_{1}=24685 \mathrm{~cm}^{-1}$ & $n=1$ & $24618 \mathrm{~cm}^{-1}$ & $24685 \mathrm{~cm}^{-1}$ \\
$v_{2}=25054 \mathrm{~cm}^{-1}$ & $n=2$ & $25000 \mathrm{~cm}^{-1}$ & $25056 \mathrm{~cm}^{-1}$ \\
$v_{3}=25131 \mathrm{~cm}^{-1}$ & $n=3$ & $25075 \mathrm{~cm}^{-1}$ & $25132 \mathrm{~cm}^{-1}$ \\
Maximum d'ab- & Position spectrale des doublets du spectre \\
sorption par ana- & \multicolumn{2}{c}{ excitonique de CuI observé sur couche } \\
lyse KK & mince selon [4].
\end{tabular}

Calcul des facteurs $\boldsymbol{f}$ des raies excitoniques. - Les facteurs $f$ des différentes raies sont calculés par la relation de Krawetz:

$$
f=\frac{m c^{2}}{\pi \mathrm{Ne}^{2}} \int n K \mathrm{~d} v,
$$

l'intégrale étant calculée sur ordinateur et les différentes bornes d'intégration étant fixées par le fond continu d'absorption. Or, ce fond continu est très sensible à la correction de phase, du moins en ce qui concerne les raies autres que $v_{1 \mathrm{~d}}$.

Dans le tableau II, on donne les facteurs $f$ calculés dans deux cas extrêmes. Dans la première ligne, les phases aux deux extrémités du spectre sont celles qui donnent le tracé optimum de la figure 2 et dans la deuxième ligne, les valeurs de $f$ sont calculées pour un profil de $n K$ obtenu sans correction de phase On remarquera que l'indétermination due à la correction de phase est inférieure à $10 \%$ pour $v_{1 \mathrm{~d}}$, mais elle peut atteindre $100 \%$ pour des raies de faible intensité qui se superposent à un fond continu important.

En évaluant le facteur $f$ global du premier doublet $v_{1 \mathrm{~d}}$, Reiss a obtenu les valeurs suivantes : $6 \times 10^{-3}$ à partir de l'absorption d'une couche mince,

TABleaU II

Tableau comparatif des valeurs des facteurs $f$ obtenus avec et sans correction de phase

$\begin{array}{lccccc} & f_{1 \mathrm{~d}} & f_{2 \mathrm{~d}} & f_{3 \mathrm{~d}} & f_{1 \mathrm{f}} & f_{2 \mathrm{f}} \\ & - & - & - & - & - \\ \begin{array}{l}\text { Avec correction de } \\ \text { phase }\end{array} & 11,54 \times 10^{-3} & 2,03 \times 10^{-3} & 0,20 \times 10^{-3} & 5,82 \times 10^{-3} & 0,32 \times 10^{-3} \\ \begin{array}{l}\text { Sans correction de } \\ \text { phase }\end{array} & 10,9 \times 10^{-3} & 1,02 \times 10^{-3} & & 2,6 \times 10^{-3}\end{array}$


$11,5 \times 10^{-3}$ par comparaison du spectre cannelé d'une couche de $0,36 \mu$ [1].

Interprétation des résultats (spectres excitoniques). a) SÉPARATION SPIN-ORBITE DE LA BANDE $\Gamma_{15}$ LA PLUS ÉLEVÉE. - La bande de valence la plus élevée est le résultat d'un mélange de fonctions d'onde $5 \mathrm{p} \mathrm{I}^{-}$ et $3 \mathrm{~d} \mathrm{Cu}^{+}$. De symétrie $\Gamma_{15}$, elle se dédouble par la séparation spin-orbite pour donner deux bandes $\Gamma_{\mathbf{8}}$ et $\Gamma_{7}, \Gamma_{8}$ étant deux fois dégénérée. Pour $\mathrm{CuBr}$ et $\mathrm{CuI}$, la bande $\Gamma_{8}$ est la plus élevée. Cette hypothèse, avancée par Cardona, a été confirmée par les calculs de Song [8].

Dans ces conditions, les deux rayons restants les plus intenses $v_{1 \mathrm{~d}}$ et $v_{1 \mathrm{f}}$ sont attribués à des transitions excitoniques respectivement à partir de $\Gamma_{8}$ et $\Gamma_{7}$. En effet, nous avons vu que les intensités d'oscillateur de ces deux raies $\left(10,9 \times 10^{-3}\right.$ ou $11,5 \times 10^{-3}$ pour $v_{1 \mathrm{~d}} ; 2,6 \times 10^{-3}$ ou $5,8 \times 10^{-3}$ pour $v_{1 \mathrm{f}}$ ) sont conformes à celles du spectre excitonique de première classe. De plus, $v_{1 \mathrm{~d}}$ est au moins égal à $2 \times f_{1 \mathrm{f}}$, ce qui confirme la dégénérescence plus élevée de $\Gamma_{8}$ par rapport à $\Gamma_{7}$.

Le spectre que nous avons mesuré sur cristal massif présente une autre particularité. L'absorption de couches minces montrait que les raies de la série $\Gamma_{8}$ sont en fait des doublets, les deux composamtes étant d'intensité comparable. Cette structure disparaît pour un cristal massif. Nous admettrons que pour la couche mince, l'effet de tensions introduites par le support entraîne une levée de dégénérescence de $\Gamma_{8}$, ce qui serait à l'origine du dédoublement des niveaux excitoniques dans ce cas. Nous verrons plus loin que la structure fine qui apparaît dans le rayon restant $v_{1 \mathrm{~d}}$ a une autre origine.

b) SÉRIes EXCITONIQues. - Pour les transitions à partir de $\Gamma_{8}$, en attribuant à $v_{2 \mathrm{~d}}$ et $v_{3 \mathrm{~d}}$ les niveaux $n=2$ et $n=3$, on obtiendrait la série hydrogénoïde suivante :

$$
v_{n}\left(\mathrm{~cm}^{-1}\right)=25190-\frac{554}{n^{2}} .
$$

La raie $v_{1 \mathrm{~d}}$ ne satisfait pas à cette loi : elle se situe à $40 \mathrm{~cm}^{-1}$ du côté des petites énergies par rapport à la position hydrogénoïde calculée. Pour $\mathrm{CuCl}$ et $\mathrm{CuBr}$, nous avons montré qu'un tel écart, respectivement de $500 \mathrm{~cm}^{-1}$ et $60 \mathrm{~cm}^{-1}$ est conforme à la théorie de Haken où le potentiel de chaque niveau excitonique est corrigé par une constante diélectrique propre.

Il est à remarquer qu'aucune des raies $v_{\mathrm{d}}$ ne correspond à l'une ou l'autre des raies désignées par $\mathrm{W}_{1}$ et $\mathrm{W}_{2}$ et observées par Cardona dans l'absorption de certaines couches minces [5].

Les intensités d'oscillateur relatives ou les facteurs $f$ constituent un autre critère pour attribuer les raies $v_{1 \mathrm{~d}}, v_{2 \mathrm{~d}}, v_{3 \mathrm{~d}}$ d'une part, les raies $v_{1 \mathrm{f}}, v_{2 \mathrm{f}}$ d'autre part, à une série excitonique de première classe.

On sait que pour une série de première classe, les facteurs $f$ varient comme :

$$
f_{n}=\frac{1}{n^{3} \varepsilon_{n}^{3}} .
$$

Cette loi ne se vérifie qu'en première approximation ; elle suppose que la série est rigoureusement hydrogénoïde, donc que la constante diélectrique est la même pour tous les niveaux. Elle suppose encore que l'énergie d'échange due à l'interaction des spins est négligeable. Dans le cas de CuI, ces deux conditions doivent être satisfaites. Nous venons de voir que l'écart par rapport à une série hydrogénoïde est faible, que d'autre part, les énergies des raies $v_{1 \mathrm{~d}}$ et $v_{1 \mathrm{f}}$ sont très différentes.

Toutefois, les informations obtenues dans ce domaine par une analyse Kramers-Krönig doivent être considérées avec prudence, surtout en ce qui concerne les raies qui se superposent à un fond continu.

Dans le tableau III, on compare les rapports théoriques des facteurs $f$ pour une série excitonique de première classe à ceux obtenus expérimentalement dans les deux cas envisagés pour l'analyse KramersKrönig.

L'accord peut être considéré comme qualitativement satisfaisant.

\section{TABLEAU III}

Tableau comparatif du rapport des facteurs $f$ des raies d'une même série excitonique

$\begin{array}{lccr} & \frac{f_{1 \mathrm{~d}}}{f_{2 \mathrm{~d}}} & \frac{f_{1 \mathrm{~d}}}{f_{3 \mathrm{~d}}} & \frac{f_{1 \mathrm{f}}}{f_{2 \mathrm{f}}} \\ & \overline{8} & \overline{27} & - \\ \begin{array}{l}\text { Valeurs théoriques } \\ \begin{array}{l}\text { Exp. avec correc- } \\ \text { tion de phase }\end{array}\end{array} & 5,6 & 57 & 18 \\ \begin{array}{l}\text { Exp. sans correc- } \\ \text { tion de phase }\end{array} & 10 & - & -\end{array}$

En particulier, on peut conclure que la raie $v_{2 \mathrm{f}}$, qui occupe une position spectrale voisine de $\mathrm{W}_{3}$ observée par Cardona et attribuée à la phase wurtzite, serait en fait une raie excitonique $n=2$ liée à la bande $\Gamma_{7}$ (ceci est d'autant plus vraisemblable que $\mathrm{W}_{1}$ et $\mathrm{W}_{2}$ qui avaient la même origine que $\mathrm{W}_{3}$ sont aussi absentes).

c) Couplage JJ. - A priori, la raie de structure fine $v_{(\mathrm{J})}$ du rayon restant $v_{1 \mathrm{~d}}$ pourrait s'interpréter de deux façons : sachant que des contraintes peuvent être à l'origine du dédoublement de $v_{1 \mathrm{~d}}$ (voir les couches minces), on pourrait admettre que la raie $v_{(\mathrm{J})}$ apparaît du fait de contraintes que subit le cristal par refroidissement, ce qui pourrait entraîner la levée de dégénérescence de $\Gamma_{8}$.

En fait, on montre que, dans le spectre de réflexion d'une intersurface CuI-quartz, $v_{(\mathrm{J})}$ peut coexister avec un rayon restant assez diffus provenant de la composante de faible énergie du doublet [13].

De plus, la raie $v_{(\mathrm{J})}$ se situe à environ $50 \mathrm{~cm}^{-1}$ de $v_{1 \mathrm{~d}}$. L'ordre de grandeur de cet écart se retrouve dans une raie analogue $\left(23889 \mathrm{~cm}^{-1}\right)$ observée dans le spectre de $\mathrm{CuBr}$ qui a été attribué à un niveau excitonique interdit $\mathbf{J}=2$. En effet, le couplage des 
spins d'un électron $\left(J=\frac{1}{2}\right)$ et d'un trou $\left(J=\frac{3}{2}\right)$ de la bande $\Gamma_{8}$ prévoit deux niveaux excitoniques, l'un avec $\mathbf{J}=1$ (transition permise), l'autre avec $\mathbf{J}=2$ (normalement interdit). Dans cette hypothèse, le niveau $J=2$ est dédoublé par le champ cristallin, l'une des deux composantes devant se décomposer en un triplet dans le champ magnétique $\mathrm{H}$. Ceci a été confirmé par les travaux de Wecker et Certier dans le cas de $\mathrm{CuBr}$ [14]. Il apparaît donc comme très probable que la raie de structure fine représente un niveau $\mathbf{J}=2$ au même titre que la raie $23889 \mathrm{~cm}^{-1}$ pour $\mathrm{CuBr}$.

d) Transitions avec PHONON. - Le rayon restant $v_{2 \mathrm{~s}}$ se traduit par un très faible maximum d'absorption vers $24210 \mathrm{~cm}^{-1}$, qui se situe donc à environ $150 \mathrm{~cm}^{-1}$ de la raie $v_{2 \mathrm{~d}}$. Cet écart est remarquable ; il est voisin du phonon longitudinal qui est de l'ordre de $150 \mathrm{~cm}^{-1}$ [15]. Par analogie avec $\mathrm{CuCl}$ et $\mathrm{CuBr}$, il est très probable que $v_{2 \mathrm{~s}}$ corresponde à une transition assistée d'un phonon longitudinal vers le niveau excitonique $v_{2 \mathrm{~d}}$. Le mécanisme est particulièrement évident pour $\mathrm{CuCl}$ où l'on peut observer jusqu'à 5 phonons [6]. Si notre interprétation se confirme pour $\mathrm{CuI}$, on devrait pouvoir mettre en évidence une structure oscillatoire dans l'absorption d'une couche mince au même titre que pour $\mathrm{CuCl}$.

Transitions à partir de bandes profondes. - A $32500 \mathrm{~cm}^{-1}$, soit environ $4 \mathrm{eV}$, apparaît une structure qui met en jeu des transitions à partir de bandes de valence ou de conduction, respectivement plus profonde ou plus élevée. Pour faciliter la comparaison de nos résultats avec les mesures de Cardona et les calculs de Song, nous avons repris, dans la mesure du possible, la notation du travail de Cardona et nous donnerons la position des bandes en $\mathrm{eV}$.

a) Aux deux maxima de réflexion $E_{1}$ et $E_{1}^{\prime}$ à $4,78 \mathrm{eV}$ et $5,11 \mathrm{eV}$, une analyse $\mathrm{KK}$ fait correspondre deux maxima d'absorption à 4,83 et $5,11 \mathrm{eV}$. Cette configuration est nettement différente des observations faites par Cardona à température ambiante, mais elle confirme un très faible palier observé par le même auteur en absorption à $77^{\circ} \mathrm{K}$ sur couche mince.

Le schéma des bandes, extrapolé de $\mathrm{CuCl}$ par Song à l'aide de la théorie de Herman et Callaway [16], [17], prévoit des transitions $\Gamma_{15}^{(2)} \rightarrow \Gamma_{1}^{(1)}$ d'énergie $5,3 \mathrm{eV}$. D'autre part, la séparation spin-orbite de $\Gamma_{15}^{(2)}$ a été calculée par Song à partir de la valeur observée pour $\Gamma_{15}^{(1)}$. La valeur obtenue est de $0,28 \mathrm{eV}$, ce qui est tout à fait comparable à la séparation $E_{1}, E_{1}^{\prime}$, soit $0,33 \mathrm{eV}$.

En prenant en considération ces deux critères : évaluation de l'énergie interbande, séparation spinorbite, Song a été amené à attribuer $E_{1}$ et $E_{1}^{\prime}$ à des transitions $\Gamma_{15}^{(2)} \rightarrow \Gamma_{1}^{(1)}$. Cette interprétation est d'autant plus justifiée qu'elle se vérifie pour les trois halogénures de $\mathrm{Cu}$.

b) La bande désignée par $E_{0}^{\prime}$ dans le travail de REVUE de PHYSique APPLIQUÉE. - T. 7, No 1, MARS 1972

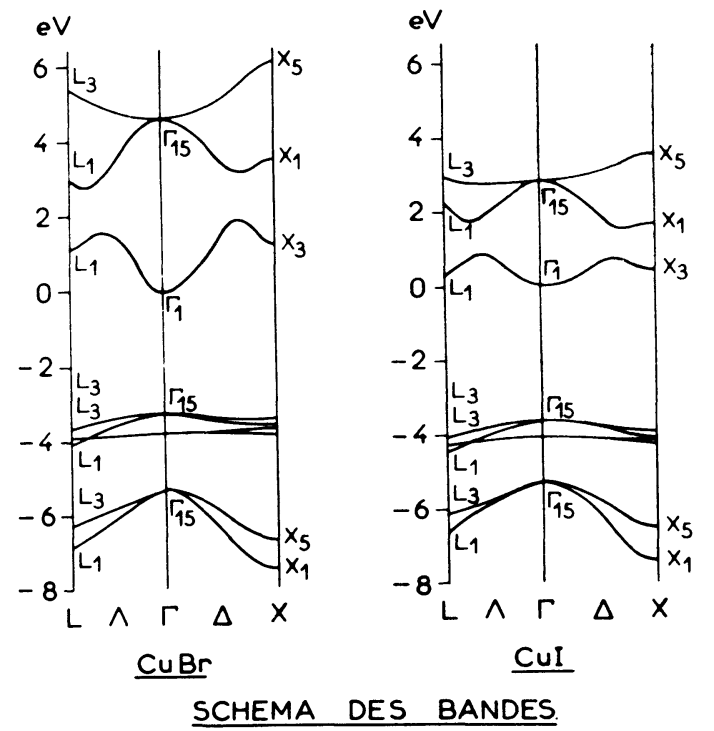

Fig. 3. - Schéma des bandes de $\mathrm{CuBr}$ et $\mathrm{CuI}$.

Cardona apparaît en doublet dans le spectre de réflexion du cristal massif.

Le maximum de réflexion que nous observons à $6,00 \mathrm{eV}$ à $9{ }^{\circ} \mathrm{K}$ se retrouve dans le spectre de réflexion sur couche mince à température ordinaire, cité par Cardona $(6,00 \mathrm{eV})$. Par contre, ce dernier spectre ne fait pas apparaître un deuxième maximum que nous observons à $6,25 \mathrm{eV}$.

Les écarts augmentent encore quand nous comparons la position spectrale des maxima du coefficient d'absorption $\mathrm{K}$ tiré d'une analyse $\mathrm{KK}$ au profil de $\mathrm{K}$ mesuré:l par absorption sur couche mince à $77^{\circ} \mathrm{K}$ par Cardona. Dans ce dernier cas, les deux composantes de $E_{0}^{\prime}$ apparaissent à 5,98 et $6,4 \mathrm{eV}$, tandis que l'analyse $\mathrm{KK}$ de notre spectre de réflexion situe les maxima respectivement à 6,07 et $6,27 \mathrm{eV}$.

Selon nos mesures, la position globale de $E_{0}^{\prime}$ serait en bon accord avec les conclusions de Song qui, selon son schéma, attribue $E_{0}^{\prime}$ à des transitions $\mathrm{L}_{3}^{(1)} \rightarrow \mathrm{L}_{1}^{(2)}$. Toutefois, nos observations ne seraient pas en accord avec la séparation spin-orbite en $L_{3}$, qui devrait être de l'ordre de $2 / 3$ de celle en $\Gamma_{15}^{(1)}$, soit $0,42 \mathrm{eV}$ [8]. Si cette valeur est en excellent accord avec la structure observée par Cardona à $77^{\circ} \mathrm{K}$ sur couche mince $(0,42 \mathrm{eV})$, elle représente le double de la séparation que nous mesurons. Il ne semble pas que cet écart provienne de l'analyse KK qui, du fait de nos hypothèses, pourrait être aberrante, surtout aux extrémités du spectre. En effet, l'écart qui apparaît est bien supérieur à celui qui sépare, dans l'ensemble du spectre, les maxima de réflexion mesurés des maxima d'absorption calculés. D'autre part, même à sa limite d'utilisation, notre montage spectrométrique ne peut entraîner une erreur de l'ordre de $2000 \mathrm{~cm}^{-1}$, soit de $80 \AA$.

Par contre, il est probable qu'aux très basses températures d'une part, avec un cristal massif d'autre part, de nouvelles structures apparaissent. En effet, 
selon e schéma de Song, des transitions, comme par exemple $\Gamma_{15}^{(1)} \rightarrow \Gamma_{15}, \mathrm{~L}_{3}^{(1)} \rightarrow \mathrm{L}_{1}^{(2)}, \mathrm{L}_{3}^{(2)} \rightarrow \mathrm{L}_{1}^{(1)}$, doivent avoir des énergies comprises entre 6 et $6,5 \mathrm{eV}$. Dans ces conditions, l'interprétation du spectre risque d'être plus compliquée.

c) Le palier, que nous pointons à $5,38 \mathrm{eV}$, n'est cité par aucun auteur. Son énergie est pratiquement la même que celle envisagée pour $\Gamma_{15}^{(2)} \rightarrow \Gamma_{1}$ dans le schéma de Song, mais nous avons vu qu'il est très probable que cette dernière transition est représentée par les pics $E_{1}$ et $E_{1}^{\prime}$. En dehors de $\Gamma_{15}^{(2)} \rightarrow \Gamma_{1}$, aucune transition entre points de symétrie élevée ne présente une énergie voisine de $5,38 \mathrm{eV}$.

Il peut paraître aléatoire d'attribuer ce palier à une certaine transition d'après la seule considération de l'énergie. En effet, il ne faut pas perdre de vue que les énergies interbandes ont été extrapolées. Pour CuI, cette extrapolation est d'autant plus critique que les énergies interbandes, aux différents points de symétrie, sont assez voisines.

Seule une étude systématique de ce palier pour les trois halogénures permettrait de trancher.

Dans l'état actuel de nos recherches, nous nous contenterons de relever qu'une transition $X_{(1)}^{1} \rightarrow X_{1}$, selon le schéma de Song, correspondrait à une énergie de l'ordre de 5,65 eV. Pour $\mathrm{CuCl}$ et $\mathrm{CuBr}$, cette transition pourrait se traduire par une absorption respectivement à $7,75 \mathrm{eV}$ et $7 \mathrm{eV}$. Les possibilités de notre montage ne nous permettent pas d'atteindre ces énergies.

Conclusions. - L'étude détaillée du spectre de réflexion d'un cristal massif à une température voisine de l'hélium liquide permet d'abord de lever des indéterminations qui sont apparues selon les auteurs dans le spectre d'absorption de couches minces.

Par exemple, aucune des raies $\mathrm{W}_{1}$ ou $\mathrm{W}_{2}$, ou encore
$\mathrm{H}_{1}, \mathrm{H}_{2}$ et $\mathrm{H}_{3}$, signalées par Cardona et attribuées à différentes structures cristallines de la couche mince, n'apparaît dans notre cas.

D'autre part, les raies excitoniques liées à la bande $\Gamma_{8}$ ne semblent plus présenter le caractère de doublet.

Un ordre de grandeur des facteurs $f$ en accord qualitatif avec des transitions excitoniques de première classe, a pu être donné en déterminant le profil d'absorption par une analyse Kramers-Krönig. On observe trois niveaux excitoniques formés avec des trous de la bande $\Gamma_{8}$, deux avec ceux de la bande $\Gamma_{7}$.

En outre, deux particularités ont été relevées :

- Le niveau $n=1$ de $\Gamma_{8}$ présente une structure fine qui peut être expliquée par le couplage $\mathbf{J J}, \mathrm{du}$ trou et de l'électron.

- Par analogie avec $\mathrm{CuCl}$ et $\mathrm{CuBr}$, un très faible maximum d'absorption peut être attribué à une transition assistée d'un phonon longitudinal.

Du côté des plus grandes énergies, notre étude met en évidence au moins trois transitions liées à des bandes plus profondes.

- Une première bande d'absorption $\left(E_{1}, E_{1}^{\prime}\right)$ est conforme aux observations de Cardona et aux calculs de Song.

- Une deuxième bande, $E_{x}$, signalée par aucun auteur, est peut-être due à des transitions au point $X$

- $\left(E_{0}, E_{0}^{\prime}\right)$ occupe une position spectrale globale conforme aux observations de Cardona, mais la séparation des deux bandes est nettement différente.

Ces transitions s'interprètent en première approximation à l'aide d'un schéma de bandes extrapolé par Song à partir de $\mathrm{CuCl}$. Un calcul détaillé de la structure des bandes, de même que l'étude du spectre de réflexion vers des énergies plus grandes seraient d'un grand intérêt.

\section{Bibliographie}

[1] Nikitine (S.), Reiss (R.) et Sieskind (M.), $C . R$. Acad. Sci., Paris, 1958, 246, 3439.

Nikitine (S.), J. Chim. Phys., 1958, 55, 621.

Nikitine (S.), Progress in Semiconductors, 1962, 6, 233.

Reiss (R.), Thèse d'Etat, Strasbourg, Cahiers de Physique, 1959, 13, 129.

[2] Nikitine (S.) et Reiss (R.), C. R. Acad. Sci., Paris, 1956, 242, 1003.

Nikitine (S.) et Reiss (R.), J. Phys. Rad., 1957, 18, 74.

[3] Ghita (L.), Tenescu (E.), Phys. Stat. Sol., 1965, 9, K. 13.

[4] Nikitine (S.), Wenger-Wursteisen (L.) et Eberhart (J. P.), Phys. Kondens. Materie, 1964, $2,355$.

[5] Cardona (M.), Phys. Rev., 1963, 129, 69.

[6] Ringeissen (J.) et Nikitine (S.), J. Physique, 1967, 28, C 3-48.

Ringeissen (J.), CoRet (A.) et Nikitine (S.), « Localized Excitations in Solids », Plenum Press, 1968 , p. 297.
[7] Nikitine (S.), LewonczuK (S.), RingeisSen (J.) et SoNG (K. S.), C. R. Acad. Sci., Paris, 1966, 262, 1506.

[8] Song (K. S.), J. Phys. Chem. Solids, 1967, 28, 2003. Song (K. S.), J. Physique, 1967, 28, 195.

[9] Goto (T.), Takahashi (T.), Ueta (M.), J. Phys. Soc. Japan, 1968, 24, 314.

[10] Satten (R. A.), Nikitine (S.), Phys. Kondens. Materie, 1963, 1, 394.

[11] Pekar (S. I.), Soviet Phys. Solid State, 1962, 4, 953.

[12] Bode (H. W.), « Network Analysis and Feedback Amplifier Design ", D. van Nostrand Inc., N. Y, 1959.

[13] SAuder (T.), Communication privée.

[14] Wecker (C.), Certier (M.) et Nikitine (S.), $J$. Physique, 1971, 32, 63.

[15] Henninger (Y.), Morlet (G.) et Hadni (A.), $J$. Physique, 1955, 26, 143.

Plendl (J. N.), Hadni (A.) et al., Appl. Optics, 1966, 5, 398.

Roth (D.), Thèse de 3e Cycle, Strasbourg, 1968.

[16] Herman (F.), J. Electron., 1955, 1, 103.

[17] Callaway (J.), J. Electron., 1957, 2, 330. 